\title{
Towards a Chatbot for educational and vocational guidance in Morocco: Chatbot E-Orientation
}

\author{
Omar Zahour $^{1}$, El Habib Benlahmar ${ }^{2}$, Ahmed Eddaoui ${ }^{3}$, Hafsa Ouchra ${ }^{4}$, Oumaima Hourrane ${ }^{5}$ \\ ${ }^{1}$ Laboratory of Information Technology and Modeling, Hassan II University Of Casablanca, Faculty of Sciences \\ Ben M'SIK,Morocco,orzahour@gmail.com \\ ${ }^{2}$ Laboratory of Information Technology and Modeling,Hassan II University Of Casablanca , Faculty of Sciences \\ Ben M'SIK,Morocco, h.benlahmer@gmail.com \\ ${ }^{3}$ Laboratory of Information Technology and Modeling,Hassan II University Of Casablanca , Faculty of Sciences \\ Ben M'SIK,Morocco, ahmed_eddaoui@yahoo.fr \\ ${ }^{4}$ Laboratory of Information Technology and Modeling, Hassan II University Of Casablanca, Faculty of Sciences \\ Ben M'SIK,Morocco,oumaima.hourrane@gmail.com \\ ${ }^{5}$ Laboratory of Information Technology and Modeling, Hassan II University Of Casablanca, Faculty of Sciences \\ Ben M'SIK,Morocco,oumaima.hourrane@gmail.com
}

\begin{abstract}
Chatbots are currently used in various online applications; often for shopping or as a personal assistant. These chatbots offer a range of potential benefits, including personalization and $24 / 7$ instant availability. These positive aspects of chatbots lend to applications in the educational sector. they represent a new type of human-machine interface in natural language. However, chatbots in academia have received only limited attention, for example by providing organizational support for studies or courses and exams. This branch of research is just emerging in the scientific community, therefore, in our article, we set up a chatbot in the field of educational and professional guidance which is based on the theory of John Holland and the RIASEC questionnaire in order to determine the dominant type of personality of undergraduate and graduate students that wants to enter the job market.
\end{abstract}

Key words: Educational and vocational guidance, John Holland theory, Natural Language Processing, Human-machine interface.

\section{INTRODUCTION}

A chatbot (or chatterbot) is software that speaks with a (human) user: it is a virtual assistant capable of answering a certain number of questions from users, providing the correct answers. Large companies have developed several chatbots for both industrial and research solutions: some of the most famous are Apple Siri, Microsoft Cortana, Facebook M, and IBM Watson. These are just some of the most popular systems. There is a wide range of less famous chatbots that have greater relevance to research and their applications, some of which will be discussed in the next chapter [1].

One of the most difficult research tasks is the development of effective chatbots: emulating human dialogues, in fact, is a really difficult task and involves problems related to the Natural Language Processing (NLP) research field [2]. Thanks to the use of NLP algorithms and techniques, it is possible to understand what the user writes and what his requests are. Generally, this task represents the heart of the system but there are a few problems: it is not possible to map all user requests, and current chatbots do not show remarkable performance due to the unpredictability of the thinking of users. users during a conversation [3]. The correct conception of the conversational flow plays an important role in the development of a chatbot. In fact, for a successful conversation, it is important to process all user requests and provide the correct answers. In the literature, we find several examples and research on the management of conversational workflow. Most of these works use ontologies, based on domain knowledge, which can be used to interpret the intentions of the user and to solve the problem of interpretation of the sentences written by the user [4].

As previously indicated, one of Chabot's applications is education and, more precisely, the field of educational and vocational guidance. Recently, there has been an increase in chatbots for e-learning platforms to support student learning. [5] Chatbot technology can be seen as an important innovation for e-learning: in fact, they are proving to be the most innovative solution to bridge the gap between technology and education. The involvement of chatbots creates an interactive learning experience for students, such as one-on-one interaction with the teacher. To test the student's behavior and in order to keep track of their improvements, bots play an essential role in improving the 
skills of an individual student. In addition, they can also play a major role in encouraging a student to work by sending out reminders and regular notifications. There are several other use cases for chatbots for e-learning, for example, it is possible to provide a system for a personalized learning experience: each student gains and absorbs things at a different rate. The use of chatbots makes it possible to adapt the speed at which a student can learn without being too arrogant [6]. The chatbot can also be used as a source of social learning, in fact, students from different backgrounds can share their views and perspectives on a specific issue while the bot can still adapt to each of them individually [7]. As is usually the case with career and vocational guidance counselors, chatbots can help these counselors in their work routine, answer students' questions concerning the various existing academic and vocational streams and the various trades as well.

This article presents the realization of a chatbot prototype to support undergraduate and graduate students during the date to make their decision on the choice of the academic and career paths. Our chatbot aims to be an e-orientation agent for students who want to know their type of personality and the trades adapted to this type according to the theory of John Holland and the RIASEC typology. The purpose of this article is to offer its users the dominant types of personality and a suitable career for this type.

\section{RELATED STUDIES}

The development of computational conversational models (chatbots) has caught the attention of AI scientists for several years. Modern intelligent conversation and dialogue systems are inspired by the principles of many disciplines, including philosophy, linguistics, computer science, and sociology [8]. This section will explore the previous work of chatbots and their implementations.

\subsection{Chatbots applications and uses}

A chatbot called ELIZA was created in 1966 at MIT to imitate human conversation to emulate a physiotherapist in clinical treatment [9]. By using keyword matching, the Chatbot aimed to encourage users to disclose information about themselves and their family members. The ALICE chatbot was created in 1995 and was inspired by ELIZA [9] [10]. ALICE was conceived as a conversationalist, and at the time, many people thought that they were talking to a real human when they interacted with him. There is a long and detailed history of chatbots that have been used in various applications, and this can be seen more widely in the literature [11].
Chatbots are now used daily by some people. Siri from Apple, Alexa from Amazon, Microsoft Cortana and Bixby from Samsung have the ability to open apps, play music, set calendar events and, overall, be a virtual assistant [12]. There were more than 30,000 text chatterbots on Facebook Messenger alone in 2017 [13] - [16]. By 2024, Global Market Insights estimates that the size of the chatbot market will exceed \$ 1.34 billion [17]. These facts and figures clearly show the rapidly growing technology of chatbots and their many uses.

\subsection{Chatbots frameworks}

Chatbot frameworks are software frameworks that provide a predefined set of functions that summarize the complexities of building a chatbot, like the NLP engine [18]. Raj describes the following chatbot frames [11]:

1. QnA Maker - a cloud-based framework provided by Microsoft that enables the development of a simple Q\&A chatbot based on FAQs, URLs, and structured documents.

2. Dialogflow - a popular cloud-based framework provided by Google that is simple to use and allows integration with many platforms.

3. Rasa NLU \& Core - an open-source framework provided for the Python development environment. It is a powerful toolbox with a steep learning curve.

4. Wit.ai - a cloud-based framework provided by Facebook that is similar to Dialogflow but not as feature-rich. It works best when integrated with Facebook Messenger.

5. Luis.ai - a cloud-based framework provided by Microsoft that has functionality similar to Dialogflow and Wit.ai.

6. Botkit.ai - similar to Rasa in that it is essentially a programming library using Javascript, but offers a graphical interface. Raj also covers in detail the construction of a chatbot using Dialogflow and the RASA stack [11].

There are several other books that cover chatbot frames and provide detailed tutorials on their use. For example, Manisha [19] discusses the Microsoft Bot Framework, Wit.ai, Dialogflow, IBM Watson and Tensorflow, a data science framework that can be used in conjunction with other frameworks to create chatbots. There is also a wide range of frameworks as alternative options.

The following table (Table 1) compares some common frameworks according to certain general parameters. These are examples of some current frameworks, but due to the rapid development in this area, they may change in the future. 
Table 1: Comparison of common chatbot Frameworks

\begin{tabular}{|l|l|l|l|l|l|l|l|l|}
\hline & Company & $\begin{array}{l}\text { Paid/Fre } \\
\text { e }\end{array}$ & $\begin{array}{l}\text { Ease of } \\
\text { use }\end{array}$ & $\begin{array}{l}\text { Out of the } \\
\text { box } \\
\text { integration }\end{array}$ & $\begin{array}{l}\text { Open } \\
\text { Source }\end{array}$ & Popularity & $\begin{array}{l}\text { Web-Bas } \\
\text { ed }\end{array}$ & Language \\
\hline $\begin{array}{l}\text { QnA } \\
\text { Maker }\end{array}$ & Microsoft & Free & High & Yes & No & Med & Yes & C\# \\
\hline Dialogflow & Google & Free & High & Yes & No & High & Yes & JavaScript \\
\hline RASA & RASA & Free & Low & No & Yes & High & No & Python \\
\hline Wit.ai & Facebook & Free & High & $\begin{array}{l}\text { Yes(Faceboo } \\
\text { k) }\end{array}$ & No & High & Yes & JavaScript \\
\hline Luis.ai & Microsoft & Free & High & Yes & No & Med & Yes & JavaScript \\
\hline Botkit.ai & Botkit & Free & Low & Yes & No & Med & No & JavaScript \\
\hline
\end{tabular}

\subsection{Common Chatbots apps}

Perhaps the most common form of chatbots is the FAQ chatbots. FAQ chatbots can be seen in many businesses as an interactive way for customers or users to gain knowledge about the business. An example is the Louis Vuitton chatbot, which is linked to Facebook Messenger1, This chatbot allows users to directly enter the chatbot to find out about a product or to select it from a predefined menu.

One of the advantages of this chatbot is its predefined menu: users are likely to click on buttons rather than typing directly on the chatbot, which reduces the number of times the chatbot is unable to return a response to the user. A link to a website is also good technical marketing, as users are likely to navigate the site, which can lead to a sale.

Chatbots whose primary purpose is to answer questions, as opposed to trying to send the user to a website, are difficult to find online. One of the reasons for this is that some companies like to maintain the illusion that the chatbot is a human answering question. Another reason is that FAQ chatbots are often not hosted on a specific web page; instead, they are connected to the Facebook page associated with the company.

There are several limits to FAQ chatbots and what they can do. One of these limitations is the need for the user to have an account for the website on which the company chooses to launch their chatbot. This specific problem concerns companies that launch their chatbot on platforms like Facebook or Slack for example. Although Facebook has more than 1.5 billion daily active users [20] - [22], it can be assumed that people who do not have a Facebook account may want answers to questions about a business. This means that while a chatbot is an interactive and interesting way to display frequently asked questions, the more traditional method of listing questions and their answers is still necessary.

Chatbots Magazine describes five limitations of Chatbots FAQs: the nature of the dialogue and the scope, scope, and

\footnotetext{
${ }^{1}$ https://www.messenger.com/t/Louis Vuitton
}

intent of typical FAQ content [23]. These include issues involving a user not expecting a chatbot, the chatbot not understanding a question, and the chatbot's location not appearing on the website.

\subsection{Natural language processing and machine learning}

Chatbots require and rely on a level of ability to understand or perform natural language processing (NLP). There is much existing textual data; from messages posted on social networks to customer-based survey data, to high-density articles. Using various text analysis techniques (such as NLP) and algorithms, text documents can be classified [24] or grouped into different grouped categories. [25] These tasks may vary from the subjects contained in the press articles to feeling pieces of revision text. This is becoming increasingly important, with a large amount of text available online, to ensure that computers can understand the human-written text.

Machine learning is a technique currently used in many applications, including extracting information from text. Machine learning involves applying pattern recognition and classification techniques in different situations. Specific algorithms and processes attempt to model human learning [26], [27]. There are many different algorithms and processes in machine learning, and the choice of which algorithm to use depends on the situation. Machine learning and NLP offer many potential areas of innovation in a university teaching environment.

\subsection{Machine learning algorithms and evaluation}

A large number of researchers are using the idea of artificial intelligence and deep learning techniques to develop chatbots with different algorithms and methods. As mentioned in [28], the authors use a repository of predefined responses and a 
model that classifies these responses to choose an appropriate response for user input. In addition, they proposed a subject-sensitive neural tensor network (TACNTN) model to classify whether or not a response is appropriate for a message. The corresponding template used to select a response for a user message. More specifically, it comprises three steps which include: pre-processing the message, retrieving response candidates from the predefined message-response pair index, then ranking the response candidates with a pre-train match model.

In [29], the authors form two word-based machine learning models, a convolutional neural network $(\mathrm{CNN})$ and a bag of SVM classifier words. The resulting scores are measured by the explanatory power index (PPE). PPE used to determine how many words contribute to the classification decision and to filter relevant information without an explicit step of extracting semantic information.

The customer service agent is a large chatbot that is used to map conversations from request to response using the sequence-to-sequence model. In addition, a sequence sequence model has two networks, one works as an encoder that maps an input sequence of variable length to a vector of fixed length, and the other works as a decoder that maps the vector to a variable length exit sequence. In [30], the authors generate word integration functionalities and form word2vec models. They formed LSTMs together with five layers and 640 memory cells using stochastic gradient descent for gradient optimization and clipping.

\section{EXPERIMENTATION AND RESULTS}

\subsection{Tool used}

In our implementation we used the DialogueFlow tool.

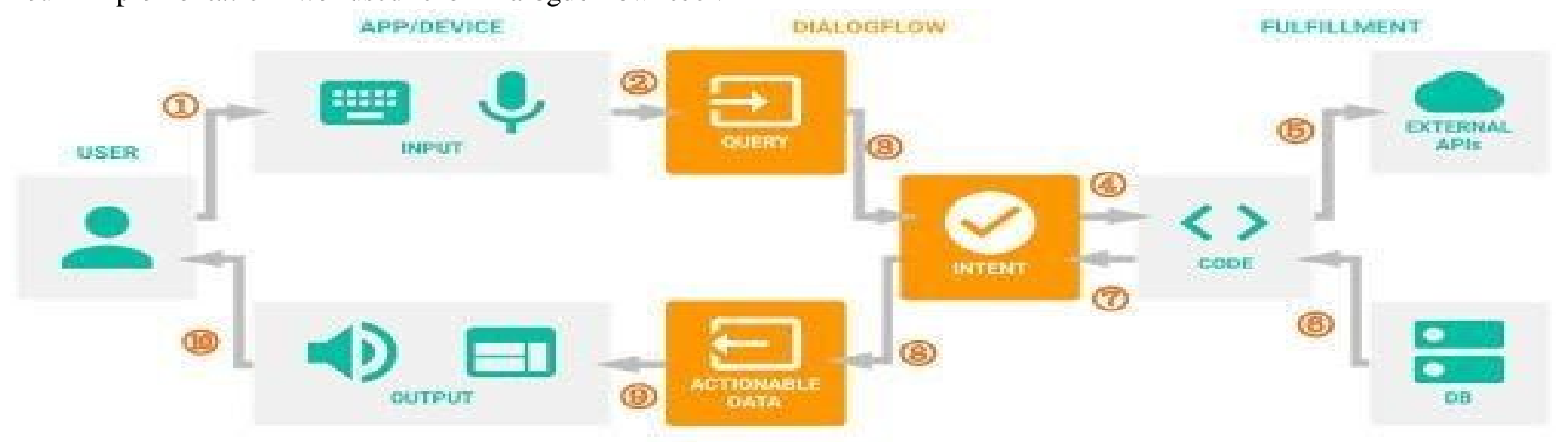

Figure 1:. The operating diagram of DialogueFlow

\subsection{The questions proposed by our chatbot}

Dialogflow (formerly called Api.ai, Speaktoit) is a Google development platform for the creation of human-computer interaction technologies based on Natural Language Processing. The company is best known for having created Assistant (by Speaktoit), a virtual assistant for Android, iOS and Windows Phone smartphones, capable of performing various tasks and answering its user's questions in natural language. Speaktoit has also created Natural Language Processing technology that integrates conversation contexts, such as exchange history, location, and user preferences.

DialogFlow is a great tool for learning how to create Chatbots which can then be integrated into your own websites or applications.

There are several good reasons to choose Dialogflow:

The price: If you only want to learn how to create a Chatbot or you don't have many users, the Standard edition is sufficient. It is completely free.

The possibility of integrating your Chatboot on different applications.

With Dialogflow, you can integrate your Chatbot with one click to the most popular instant messaging applications such as Facebook Messenger, Slack, Twitter, Kik, Line, Skype, Telegram, Twilio and Viber. It will also work on some voice assistants like Google Assistant, Amazon Alexa or Microsoft Cortana.

Natural Language Processing: Compared to other platforms that use predetermined questions like Chatfuel, Dialogflow offers an excellent user experience thanks to Natural Language Processing.

This figure shows how Dialogue flow works: 
The questions proposed by our chatbot are about 301 questions in French language which concern the educational and professional orientation according to the model of John Holland following the RIASEC typology, these questions are distributed according to four categories namely: Activities, Skills, The occupations, The personality. Each category of questions contains Six sub-categories: Realistic, Investigator, Artistic, Social, Entrepreneur, and Conventional.
According to the user's responses, the end goal of our chatbot is to allows him to determine the dominant type of his personality.

\subsection{The result obtained}

In this section, the following figures show the results obtained from the different communication windows of our chatbot.

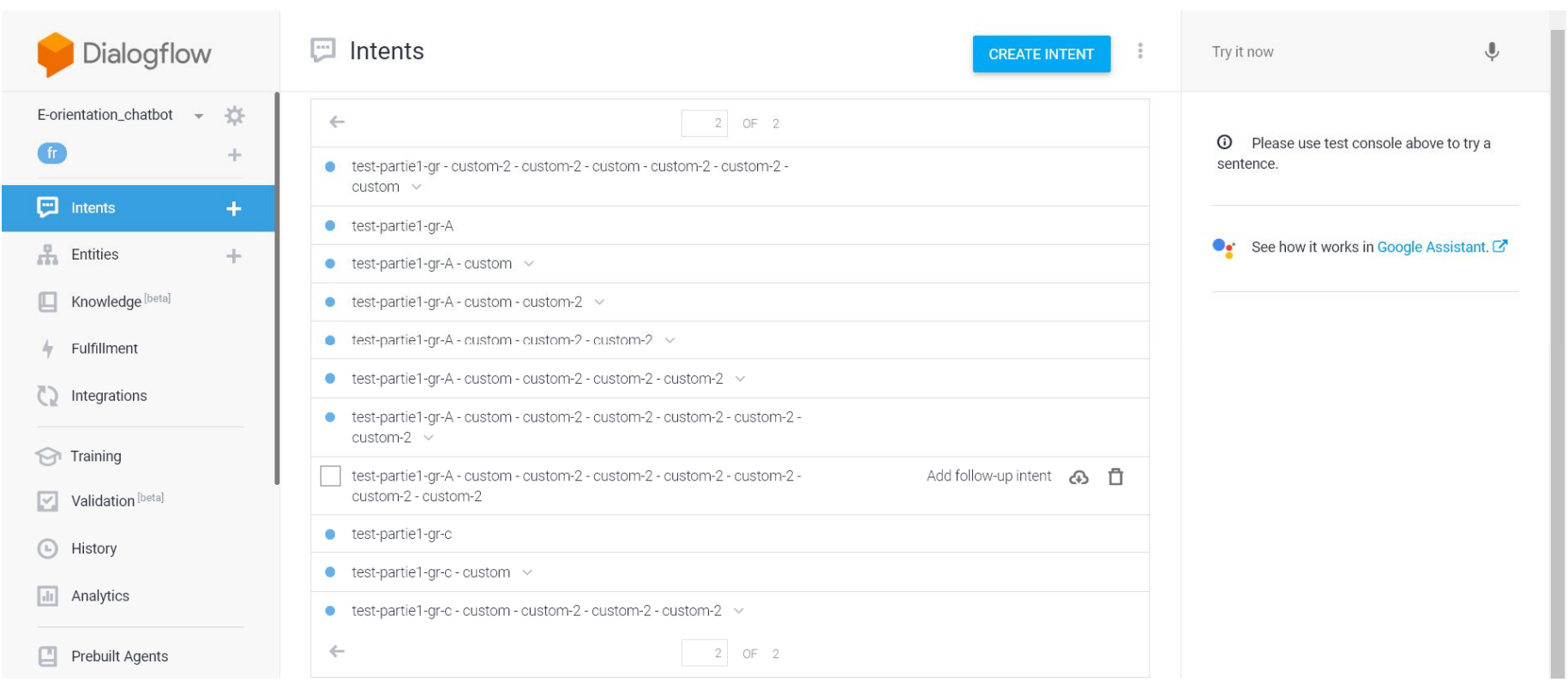

Figure.2: Diagram of DialogueFlow showing the intents of E-orientation Chatbot

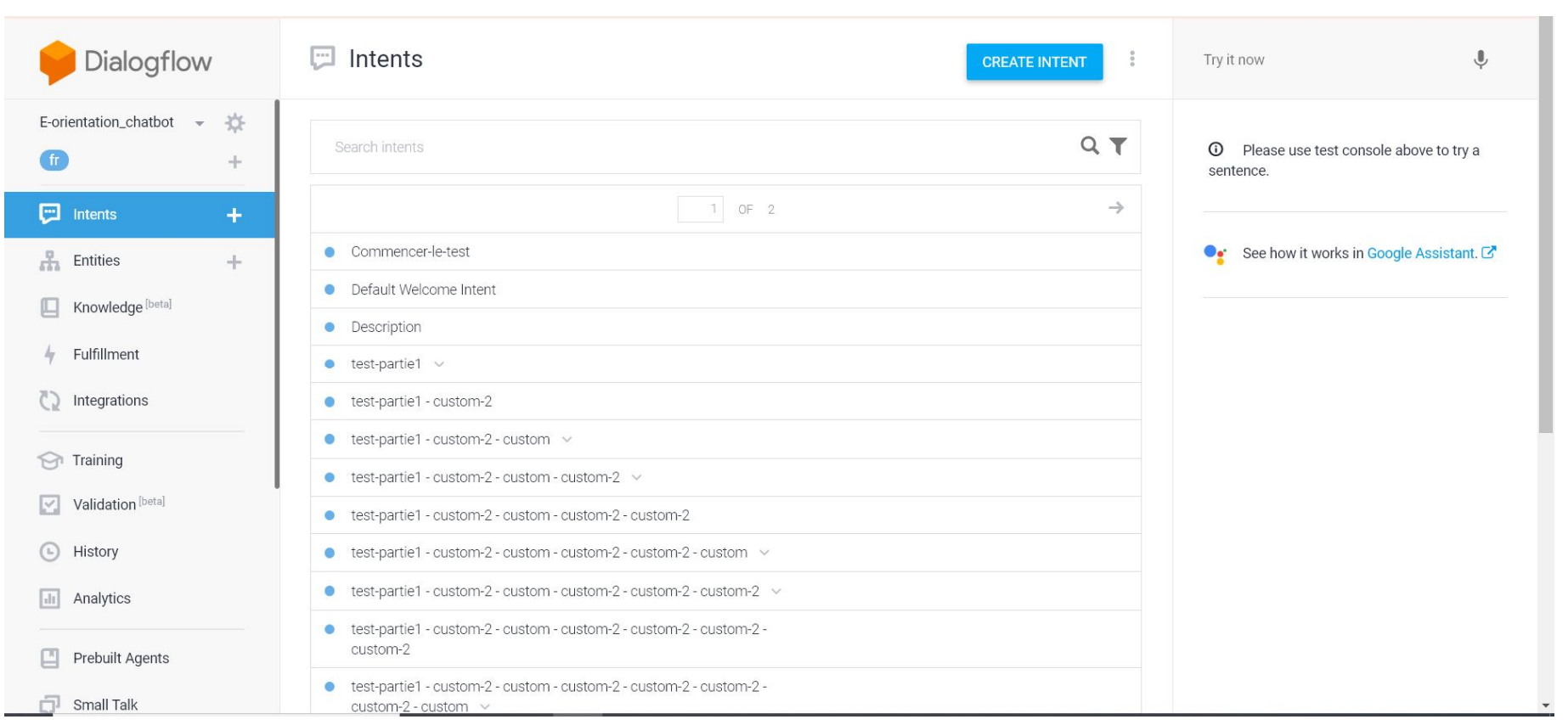


Omar Zahour et al., International Journal of Advanced Trends in Computer Science and Engineering, 9(2), March - April 2020, 2479 - 2487

Dialogflow

E-orientation_chatbot $\rightarrow$ 就

(fr)

Intents $\quad+$

혼 Entities

$+$

(17) Knowledge ${ }^{[\text {beta] }}$

4. Fulfillment

C. Integrations

Q. Training

V. Validation [beta]

(ㄴ) History

(1ili) Analytics

미 Prebuilt Agents

[ᄆ] Small Talk mantents

test-partie1 - custom-2 - custom - custom-2 - custom-2 - custom

- test-partie1 - custom-2 - custom - custom-2 - custom-2 - custom-2

- test-partie1 - custom-2 - custom - custom-2 - custom-2 - custom-2 custom-2

- test-partie 1 - custom-2 - custom - custom-2 - custom-2 - custom-2 custom-2 - custom $、$

- test-partie 1 - custom-2 - custom - custom-2 - custom-2 - custom-2 custom-2 - custom - custom-2

- Lest-pal lie1-yi

- test-partie1-gr - custom-2

- test-partie1-gr - custom-2 - custom-2

- test-partie1-gr - custom-2 - custom-2 - custom

- test-partie 1-gr - custom-2 - custom-2 - custom - custom-2

- test-partie1-gr - custom-2 - custom-2 - custom - custom-2 - custom custom

- test-partie1-gr - custom-2 - custom-2 - custom - custom-2 - custom-2

Figure 3: Diagram of DialogueFlow showing the intents of E-orientation Chatbot
- test-partie1

Enter name

+ New parameter
Try it now

(i) Please use test console above to try a sentence.

-9. See how it works in Google Assistant. ¿

$\begin{array}{ll}\square & \text { Enter name Enter entity } \quad \text { Enter value } \\ + \text { New parameter } & \end{array}$

\section{Responses ?}

DEFAULT FACEBOOK MESSENGER +

Text Response

(2) 0

1 Donc, voici la liste des activités du premier groupe $\mathrm{R}$

2 Enter a text response variant

ADD RESPONSES

Set this intent as end of conversation 
- test-partie1

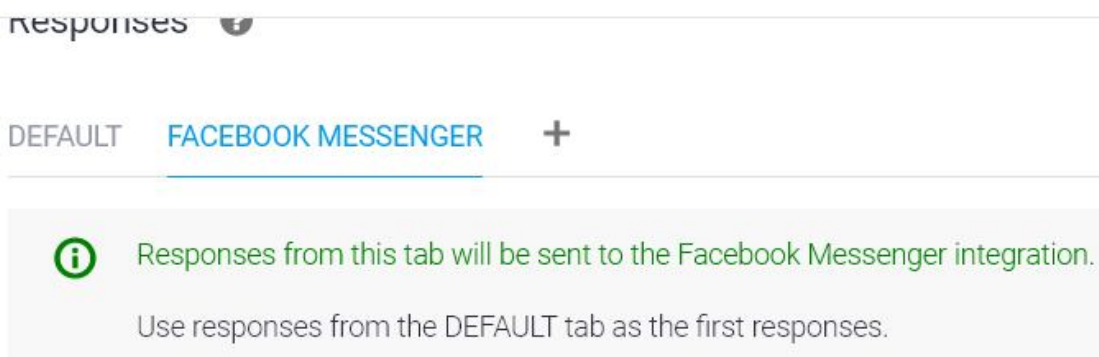

(i) Responses from this tab will be sent to the Facebook Messenger integration. Use responses from the DEFAULT tab as the first responses.

Quick Replies (? $\mathbf{0}$
1. Planter, entretenir des arbres, des arbus
Oui
Non
ADD RESPONSES
D Set this intent as end of conversation ?

Figure 4: Diagram showing the defined responses of E-orientation Chatbot

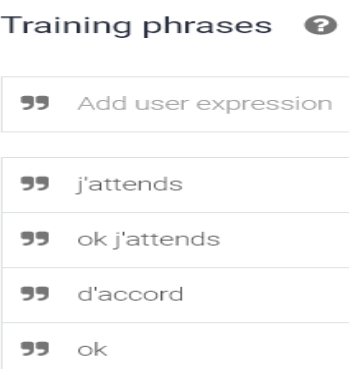

Figure 5: Diagram showing the training phrases 


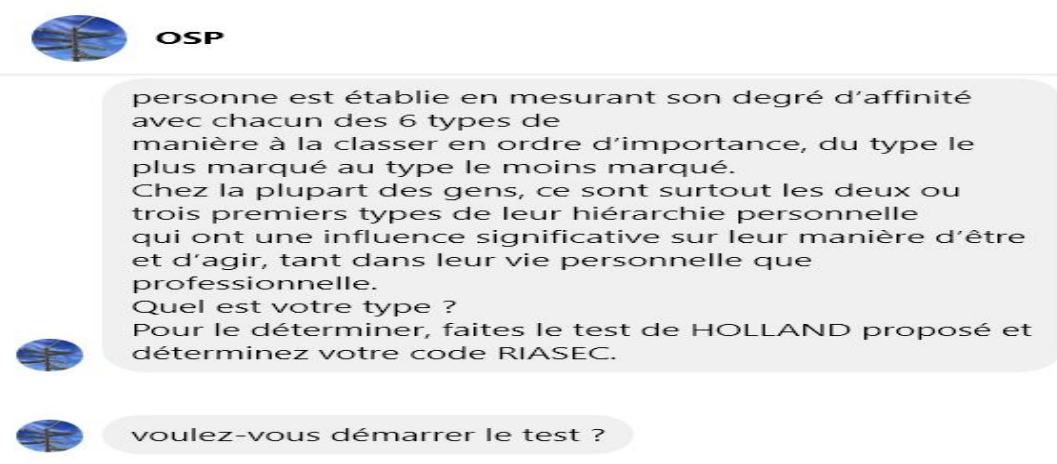

Bravo, vous avez réponder pour toutes les 15 activités du premier groupe $\mathrm{R}$.

Voulez-vous continuer votre test pour passer au deuxiéme groupe pour répondez à chaque activités selon votre gout?

Bien, alors voici les 15 questions du deuxiéme groupe " I

" de la premiére partie " Interets et activités "

1. Lire des revues scientifiques ou spécialisées.

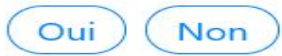

Figure 6: Diagram showing the conversations of E-orientation Chatbot in the Facebook page

We integrated our Chatbot into a Facebook page which is OSP,

https://www.facebook.com/OSP-852490914906981/?ref=ay mt_homepage_panel\&eid=ARAzi_iNzBufXDF4siAMBF7k oJGcahqJWz6skM99-0VbP97fX7_vOZ8zEZzmj5cB3u5JLU mI5Hf83kgB

\section{CONCLUSION}

In our previous work we have made a classification of educational and vocational guidance questions according to Holland's theory using the neural network methods and the BERT method too [31], [32]. In this article, an original approach to a chatbot was introduced. In particular, the proposed system is based on an online orientation platform for university students and also for graduate students who seek to integrate the job market by finding the job suited to their preferences. A real case was studied by developing a Chatbot for students of the Master Bigdata and Datascience cycle of the Ben M'Sik Faculty of Sciences, Hassan II University in Casablanca, Maroc. The results obtained by the experimental campaign are satisfactory and show the good perspective of this type of approach. Subsequent developments involve the application of the proposed approach in various contexts and an improvement of the online educational and vocational guidance platform.

\section{CONFLICT OF INTEREST}

The authors declare that there is no conflict of interest.

\section{REFERENCES}

[1]D'Aniello, G., Gaeta, M., Orciuoli, F., Tomasiello, S., Loia, V.: "A dialogue-based approach enhanced with situation awareness and reinforcement learning for ubiquitous access to linked data." In: Proceedings - 2014 International Conference on Intelligent N, pp. 249-256 (2014). https://doi. org/10. 1109/incos. 2014. 73. A. no. 7057098

[2] V . B. a C." with serverless computing". I. P. of the 1st I. W. on M. of T. and Api. (2016). A. no. 5 Yan, M., Castro, P., Cheng, P., Ishakian

[3]Bi, Y., Deng, K., Cheng, J.:" A keyword-based method for measuring sentence similarity." In:Proceedings of the 2017 ACM on Web Science Conference, pp. 379-380 (2017) 
[4] Lin, L., D’Haro, L.F., Banchs, R.: "A Web-based platform for collection of human-chatbot interactions." In: Proceedings of the Fourth International Conference on Human Agent Interaction, pp. 363-366 (2016)

[5]D'Aniello, G., Gaeta, A., Gaeta, M., Tomasiello, S.: "Self-regulated learning with approximate reasoning and situation awareness." J. Ambient Intell. Hum. Comput. 9, $1-14(2016)$

[6]H. M. ." A. chatbot using L. multi-layer embedding for elderly care." I. I. C. on O. T. (ICOT) (2017) Su, M.H., Wu, C.H., Huang, K.Y., Hong, Q.B., Wang

[7] Hussain, S., Athula, G. . E. "a conventional chatbot knowledge base to external knowledge source and introducing user based sessions for diabetes education". I. 32nd I. C. on A. I. N. and A. W. (WAINA) (2018)

[8]S. Oraby, P. Gundecha, J. Mahmud, M. Bhuiyan, and R. Akkiraju, "Modeling Twitter Customer ServiceConversations Using Fine-Grained Dialogue Acts," in Proceedings of the 22nd International Conference on Intelligent User Interfaces - IUI "17, 2017, pp. 343-355

[9]B. Abu Shawar and E. Atwell, "Chatbots: are they really useful?," LDV-Forum Zeitschrift für Comput. und Sprachtechnologie, 2007.

[10] B. A. Shawar and E. Atwell, "ALICE chatbot: Trials and outputs," Comput. y Sist., 2015.

[11] S. Raj, Sumit Raj - Building Chatbots with Python Using Natural Language Processing and Machine Learning-Apress (2019). 2018.

[12] R. Dale, "The return of the chatbots," Nat. Lang. Eng., 2016.

[13] P. B. Brandtzaeg and A. Følstad, "Why people use chatbots," in Lecture Notes in Computer Science (including subseries Lecture Notes in Artificial Intelligence and Lecture Notes in Bioinformatics), 2017.

[14] A. Følstad and P. B. Brandtzaeg, "Chatbots and the New World of HCI," Interactions, 2017.

[15] P. B. Brandtzaeg and A. Følstad, "Chatbots," Interactions, 2018.

[16] P. B. Brandtzaeg and A. Følstad, "Chatbots: User changing needs and motivations," Interactions, 2018.

[17] Future Market Insights, "Macadamia Market - Global Industry Analysis, Size and Forecast, 2018 to 2028," Futur. Mark. Insights, 2018.

[18] Maruti Techlabs, "How To Develop a Chatbot From Scratch - Chatbots Magazine," Chatbots Mag., 2017.

[19] G. Biswas, R. S. Baker, and L. Paquette, "Data Mining Methods for Assessing Self-Regulated Learning," in Handbook of Self-Regulation of Learning and Performance, 2019.

[20] Statista, "Number of mobile phone users worldwide from 2015 to 2020 (in billions)," Number Mob. phone users Worldw. from 2015 to 2020 (in billions), 2018.

[21] Statista, "Twitter: number of active users 2010-2019 Statista," Twitter: number of active users, 2019. .

[22] Statista, "Number of social media users worldwide 2010-2021 (in billions)," The Statistics Portal, 2018. .

[23] M. Y. Kim and R. Goebel, "Two-step cascaded textual entailment for legal bar exam question answering," in Proceedings of the International Conference on Artificial Intelligence and Law, 2017.

[24] T. Zhao, H. Liu, K. Roeder, J. Lafferty, and L. Wasserman, "The huge package for high-dimensional undirected graph estimation in R," J. Mach. Learn. Res., 2012.

[25] V. Mijangos, G. Sierra, and A. Montes, "Sentence level matrix representation for document spectral clustering," Pattern Recognit. Lett., 2017.

[26] P. Langley, "The changing science of machine learning," Machine Learning. 2011.

[27] A. L. Blum and P. Langley, "Selection of relevant features and examples in machine learning," Artif. Intell., 1997.
[28] Y. Wu, Z. Li, W. Wu, and M. Zhou, "Response selection, with topic clues for retrieval-based chatbots," Neurocomputing, vol. 316, pp. 251-261, N. 2018

[29] L. Arras, F. Horn, G. Montavon, K.-R. Müller, and W. Samek, "\&quot; What is relevant in a text document?\&quot;: An interpretable machine learning approach," PLoS One, vol. 12, no. 8, p. e0181142, A. 2017

[30] A. Xu, Z. Liu, Y. Guo, V. Sinha, and R. Akkiraju, "A New Chatbot for Customer Service on Social Media," in Proceedings of the 2017 CHI Conference on Human Factors in Computing Systems - CHI "17, 2017, pp. 3506-3510.

[31] Omar Zahour, El Habib Benlahmar, Ahmed Eddaoui, Oumaima Hourrane "Automatic Classification of Academic and Vocational Guidance Questions using Multiclass Neural Network " January 2019, International Journal of Advanced Computer Science and Applications 10(10);

DOI: 10.14569/IJACSA.2019.0101072

[32] Omar Zahour, El Habib Benlahmar, Ahmed Eddaoui, Oumaima Hourrane "towards a system for predicting the category of educational and vocational guidance questions using bidirectional encoder representations of transformers (BERT)", Volume 9,No.1,January-February2020, International Journal of Advanced trends in computer science and
engineering,Available http://www.warse.org/IJATCSE/static/pdf/file/ijatcse69 912020.pdf https://doi.org/10.30534/ijatcse/2020/69912020 